\title{
2012 AAES Engineering Salaries Update Presents the State of the Profession
}

In reflection of a relatively stagnant economy, U.S. engineers' compensation appears to have plateaued in the most recent American Association of Engineering Societies (AAES) research. The findings presented in Engineers' Salaries: Special Industry Report present engineers' salaries as of February 1, 2012, and thus are unaffected by any recent improvements in U.S. economic activity.

The survey, conducted by the Engineering Workforce Commission (EWC), collected salaries and categorized the data according to discipline, levels of experience, supervisory responsibility, education, and industry sectors. The report also utilized geographic location and the size of employing company as additional classifying factors.

On a generalized scale, the 2012 data does not vary significantly from the salaries reported in 2011, with a slight downtick in median earnings for more experienced workers and a slight uptick for those just starting out in the field.

Of all 29,898 survey respondents in 2012, those with no experience reported a median salary of $\$ 63,858$, up from $\$ 58,354$ in 2011 . For $13-16$ years of experience, that median rose to $\$ 90,290$, a slight decrease from 2011's $\$ 91,708$, while the reported salary for a worker with 35 years of experience or more remained unchanged at $\$ 103,397$.

For more specific information, see Table I for salaries based on discipline, Table II for salaries based on supervisory responsibility and level of education, and Table III for salaries based on employer.

This marks the $46^{\text {th }}$ EWC survey of U.S. engineers' compensation. To purchase the full report, visit the EWC website at www.ewc-online.org.

\begin{tabular}{|c|c|c|c|c|c|c|}
\hline \multicolumn{7}{|c|}{ Table I. Median Annual Salaries for Engineers by Discipline (in Dollars) } \\
\hline \multirow[b]{2}{*}{ Discipline } & \multicolumn{6}{|c|}{ Number of Years after B.S. } \\
\hline & 0 & 5 & $9-10$ & $13-16$ & $21-25$ & $35+$ \\
\hline Aerospace Engineers & - & - & 90,997 & 104,415 & 105,927 & 137,530 \\
\hline Computer Engineers & - & 68,782 & 79,605 & 92,255 & 104,393 & 122,589 \\
\hline Chemical Engineers & - & 68,754 & 81,422 & 88,857 & 101,074 & 110,874 \\
\hline Civil Engineers & 58,243 & 64,628 & 72,328 & 82,994 & 92,844 & 101,790 \\
\hline Electrical Engineers & 62,321 & 75,429 & 85,180 & 94,064 & 105,938 & 113,214 \\
\hline Engineering Science & - & 96,824 & 91,160 & 86,109 & 94,467 & 83,442 \\
\hline Environmental Engineers & 51,002 & 64,673 & 81,004 & 88,264 & 107,213 & 107,120 \\
\hline General Engineers & - & 78,750 & 88,233 & 96,492 & 103,284 & 92,999 \\
\hline Industrial Engineers & - & - & 76,130 & 86,113 & 94,724 & 108,646 \\
\hline Materials Engineers & - & - & 93,568 & 100,354 & 111,350 & 135,711 \\
\hline Mechanical Engineers & 56,208 & 76,937 & 85,073 & 94,394 & 104,229 & 119,524 \\
\hline Nuclear Engineers & - & - & - & 99,334 & 105,192 & 150,953 \\
\hline
\end{tabular}

Table II. Median Annual Salaries for Engineers Based on Experience, Supervisory Responsibility, and Level of Education (in Dollars)

\begin{tabular}{|c|c|c|c|c|c|c|}
\hline \multirow[b]{2}{*}{ Employer } & \multicolumn{6}{|c|}{ Number of Years after B.S. } \\
\hline & 0 & 5 & $9-10$ & $13-16$ & $21-25$ & $35+$ \\
\hline \multicolumn{7}{|c|}{ Nonsupervisory } \\
\hline B.S. & 63,858 & 73,100 & 78,227 & 85,470 & 94,508 & 94,719 \\
\hline M.S. & - & 75,937 & 87,218 & 94,749 & 101,378 & 102,408 \\
\hline Ph.D. & - & - & 95,705 & 102,451 & 112,088 & 143,857 \\
\hline \multicolumn{7}{|l|}{ Supervisory } \\
\hline B.S. & - & - & 85,765 & 92,308 & 103,754 & 116,321 \\
\hline M.S. & - & 119,998 & 111,714 & 120,281 & 128,075 & 127,795 \\
\hline Ph.D. & - & - & - & 126,758 & 139,927 & 162,764 \\
\hline
\end{tabular}

Table III. Median Annual Salaries for Engineers Based on Experience and Employer (in Dollars)

\section{Employer}

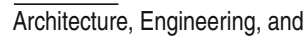
Related Services

Primary Metal Mfg.

Fabricated Metal Product Mfg.

Public Administration

Motor Vehicle Mfg.

Professional, Scientific, and

Technical Services

R\&D Organizations

Utilities

Electric Power Generation

Transmission \& Distribution

Mining

Machinery Mfg

Transportation Equipment Mfg.

Transportation and Warehousing

All Nonmanufacturing

All Mfg. Number of Years after B.S.

\begin{tabular}{|c|c|c|c|c|c|}
\hline 0 & 5 & $9-10$ & $13-16$ & $21-25$ & $35+$ \\
\hline 56,375 & 69,087 & 82,246 & 98,193 & 109,883 & 130,625 \\
\hline- & - & - & 88,584 & 92,943 & 109,163 \\
\hline- & - & 73,931 & 77,667 & 85,617 & 88,154 \\
\hline 55,742 & 66,175 & 70,583 & 82,162 & 94,449 & 92,209 \\
\hline 62,321 & 79,296 & 96,014 & 99,664 & 101,948 & 100,189 \\
\hline 54,340 & 69,487 & 84,083 & 95,201 & 108,576 & 134,366 \\
\hline 52,000 & 72,105 & 84,951 & 95,331 & 111,278 & 139,732 \\
\hline 64,676 & 74,826 & 79,161 & 91,059 & 111,741 & 126,545 \\
\hline 62,678 & 74,380 & 75,655 & 87,488 & 110,041 & 124,990 \\
\hline- & 77,272 & 86,471 & 98,901 & 115,676 & 128,093 \\
\hline- & - & 74,932 & 82,423 & 90,737 & 86,004 \\
\hline 61,778 & 79,056 & 94,540 & 99,332 & 101,258 & 100,189 \\
\hline 53,601 & 65,217 & 73,231 & 82,930 & 88,910 & 96,297 \\
\hline 54,340 & 69,481 & 78,433 & 88,115 & 98,062 & 110,899 \\
\hline 66,313 & 78,300 & 87,929 & 96,247 & 106,920 & 112,507 \\
\hline
\end{tabular}

